\title{
Arquitectura de procesos para las instituciones públicas de educación superior
}

DOI: 10.22403/UQROOMX/TYP01/08

Adalberto Velázquez Méndez

4

Armando Maldonado Talamantes

RESUMEN

La arquitectura de procesos es un elemento esencial del modelo de negocios de toda organización, particularmente para una Institución de Educación Superior (IES). En este trabajo se propone una especificación de la arquitectura de procesos de una IES, que puede ser utilizado como marco de referencia en las tareas de reingeniería o rediseño de sus procesos esenciales. Este modelo permite incorporar la estrategia institucional en los procesos de negocio de la IES. Los responsables de la estrategia de negocios definen objetivos, metas y acciones, que deberán estar contemplados en los procesos esenciales de la organización, asegurando así que sean considerados en la estrategia de Tecnologías de Información (TI), diseñada para soportar dichos procesos con la incorporación de las Tı. Contar con una arquitectura de procesos, desde una perspectiva sistémica, adquiere entonces gran relevancia, pues comprende una especificación conceptual de la estrategia de la IES sobre la cual se sustentan sus servicios de información.

\begin{tabular}{r|l} 
Palabras & $\begin{array}{l}\text { Arquitectura de procesos, modelo de negocios, especificación } \\
\text { de procesos y actividades, servicios de información, institución } \\
\text { de educación superior. }\end{array}$
\end{tabular}

"Universidad de Quintana Roo / avelazquezm@correo.uqroo.mx

*kinstituto Tecnológico Autónomo de México / armando@itam.mx 
Arquitectura de procesos

para las instituciones públicas

de educación superior

\section{Antecedentes}

Con la aparición de la internet, las instituciones de educación superior (IES) se han replanteado los retos que venían enfrentado, en particular, el correspondiente al soporte de sus procesos fundamentales (críticos) de negocio, a través de servicios de información, es decir, la administración eficiente del componente información implícito en sus procesos fundamentales de Docencia, Investigación, Extensión y Difusión.

Pocas son las instituciones que han tenido éxito en esta tarea, más aún, existen casos ya reportados en que las IES han abandonado proyectos ambiciosos, cancelando iniciativas de desarrollo y replanteando sus requerimientos en una solución más modesta y viable. ¿Qué está fallando? La respuesta no es simple pues son varios los factores en juego, siendo los más importantes:

I) Poca (o nula) alineación entre la estrategia institucional (Plan Estratégico de Desarrollo Institucional, PLADES) y la estrategia de Tecnologías de la Información (TI) (Henderson, 1992) en las IES.

2) Las TI se conciben como la solución a la problemática institucional, y no como un facilitador de los procesos institucionales. Se confunden los medios con los fines.

3) Se emprenden iniciativas de desarrollos tecnológicos sin un rediseño de los procesos fundamentales, o más aún, sin una reingeniería de los procesos de negocio de las IES. Si automatizamos un proceso "enfermo" (que requiere ser rediseñado), sólo obtendremos los mismos errores, pero más "eficientemente".

4) Se considera la tecnología como un gasto y no como una inversión que las IES requieren para poder seguir compitiendo en igualdad de circunstancias, o con ventaja, en el mercado de la educación superior.

\section{Metodología}

La arquitectura de procesos comprende el conjunto de procesos esenciales de la empresa, sus relaciones entre sí y con algunos procesos de soporte y de administración (Maldonado, 2003). Constituye el portafolio de procesos 
de la empresa y una representación (o modelado) detallada de las actividades que se realizan en ellos.

¿Cómo obtener la arquitectura de procesos? Mediante cuatro pasos:

I) Se documenta la estructura organizacional

2) Se identifica o definen las funciones (actividades) de negocio, primarias y secundarias, de la empresa.

3) Se define el diagrama de la vista horizontal

4) Se realiza el mapeo del diagrama de configuración de valor al diagrama de la arquitectura de procesos.

\section{Estructura organizacional}

Para que este modelo pueda ser adoptado por las IES que así lo requieran, se incluye en esta sección la Estructura Organizacional de una IES genérica (véase figura I), con base en un modelo departamental.

Las unidades organizacionales de esta IES genérica están agrupadas en un modelo institucional mínimo que contempla sólo a las unidades organizacionales directamente relacionadas con las funciones sustantivas de Docencia, Extensión e Investigación.

RECTORÍA

I) A cargo del gobierno de la IES

2) Generador de las líneas estratégicas institucionales que comprende el Plan de Desarrollo Institucional (PLADES) y el Programa Integral de Fortalecimiento Institucional (PIFI)

División ACADÉmICA

3) Corresponde a un área de conocimiento

4) A cargo de la administración de los programas académicos (carreras) pertenecientes al área de conocimiento

5) Agrupa a varios departamentos académicos Departamento Académico

6) Corresponde a un área de especialidad

7) A cargo de la administración de las materias y programas de estudio (contenidos)

8) Responsable de la impartición de cursos correspondientes a los programas académicos 
Arquitectura de procesos

para las instituciones públicas

de educación superior

A1 112

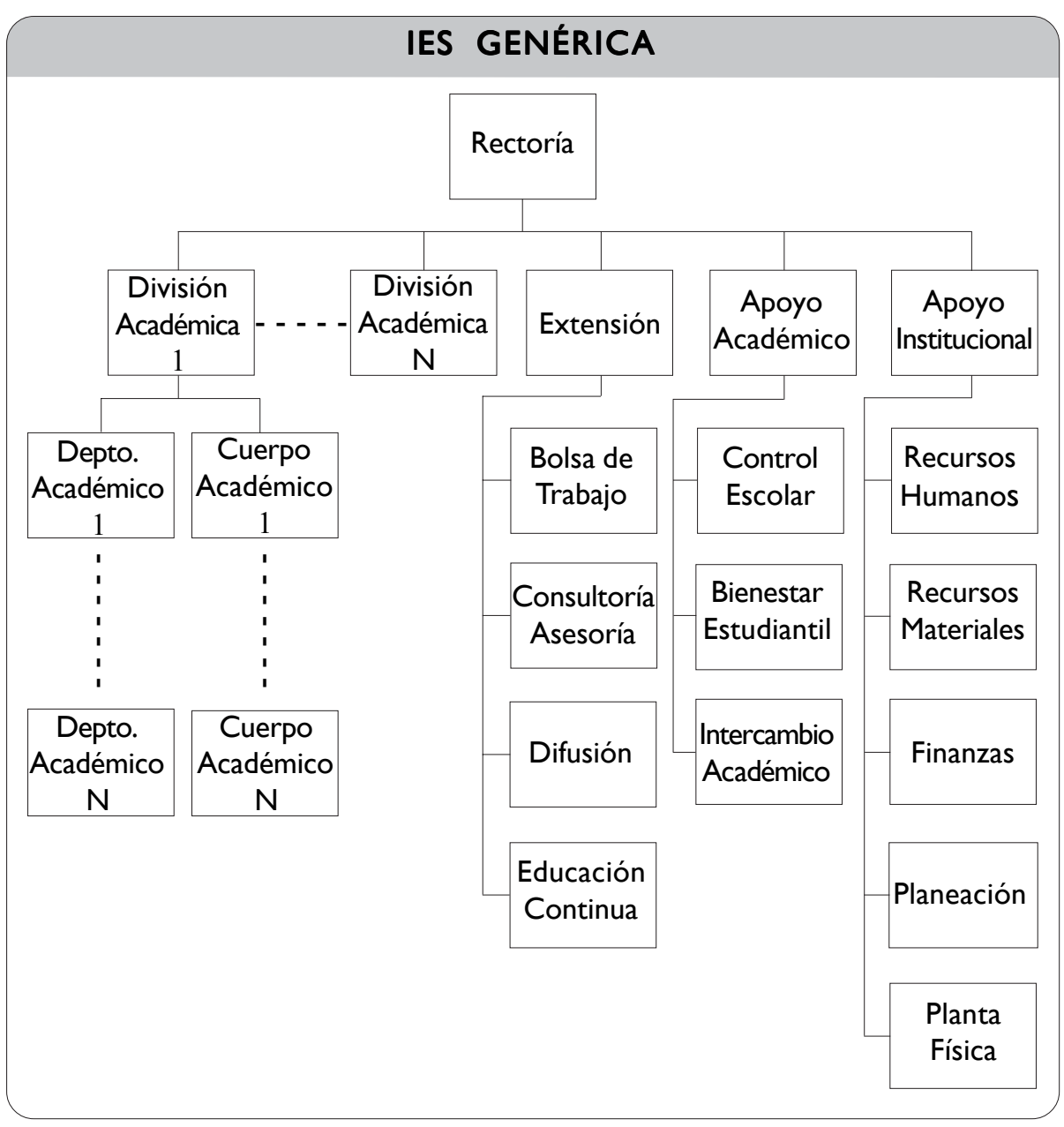

Figura I. Estructura Organizacional de una IES. 
9) A cargo de la administración de la planta de profesores (por área de especialidad)

10) Responsable de la función de docencia

Cuerpo ACAdÉmico

II) Corresponde a una Línea General de Aplicación del Conocimiento (LGAC)

12) Agrupa a investigadores en temáticas asociadas con la LGAC

13) Responsables de la función de investigación

EXTENSIÓN

14) Responsable de la difusión de los productos de la investigación

15) Responsable de la difusión de los productos de la función docencia (egresados) mediante la Bolsa de Trabajo

16) Administra la aplicación del conocimiento (producto de la investigación), mediante consultorías y asesorías especializadas

17) A cargo de las actividades de educación continua

18) Responsable de la función de extensión

ApOYo ACADÉmICO

19) A cargo de las actividades de soporte a los procesos fundamentales de docencia e investigación

20) Responsable de la promoción de los programas académicos de la institución y del proceso de selección de aspirantes

21) Responsable del registro académico de los alumnos, inscripciones, calificaciones, titulación, cambios de situación académica (bajas, cambios de carrera, etcétera)

22) Responsables de las acciones encaminadas al bienestar estudiantil: servicio social, becas, servicios médicos y psicológicos, actividades culturales y deportivas, etcétera

APOYO INSTITUCIONAL

23) A cargo de la administración de los recursos humanos, materiales y financieros que requieren las funciones sustantivas

24) A cargo de la salvaguarda de los bienes de la institución

25) A cargo de la realización del Programa Operativo Anual (POA) de la Institución

26) Responsables de la realización del Programa Integral de Fortalecimiento Institucional (PIFI), en coordinación con las unidades organizacionales académicas y administrativas de la IES 
Arquitectura de procesos

para las instituciones públicas

de educación superior

\section{Funciones y actividades}

Con el propósito de que el modelo propuesto pueda ser utilizado como marco de referencia por las IES que requieran de una especificación formal de sus procesos de negocio, se presentan a continuación las actividades más comunes de las funciones sustantivas y adjetivas de una IES genérica.

Funciones sustantivas

DOCENCIA. Comprende los programas y actividades de instrucción y transmisión del conocimiento, formalmente organizados por las IES, que se ofrecen como cursos y tutorías académicas, independientemente de que por ellos se otorgue o no un grado académico y de que sean estudios no escolarizados

INVESTIGACIÓN. Integra las acciones, proyectos y programas de la IES, orientados de manera específica a la generación de conocimiento y su aplicación innovadora.

EXTENSIÓN. Conformada por actividades y programas que tienen como objetivo vincular a la institución con su entorno, y extender a la comunidad los beneficios de la cultura mediante la difusión, divulgación y promoción del conocimiento científico, tecnológico, artístico y humanístico.

\section{Funciones adjetivas}

APOYO ACADÉMICO. Comprende las acciones y programas orientados a mejorar la calidad y eficiencia de las funciones sustantivas de la institución (docencia, investigación y extensión), que benefician directamente a estudiantes $y$ académicos.

APOYO INSTITUCIONAL. Destinada a asegurar la disposición permanente y oportuna de los recursos humanos, materiales y financieros necesarios para la operación del sistema. Esta categoría incluye las actividades de la administración central y unidades de apoyo administrativo de la institución, tendientes a la planeación, organización, dirección y control.

AdMINISTRACIÓN DE LA PLANTA FísICA. Conformada por actividades y programas destinados a la administración, operación, conservación, mantenimiento, expansión y ampliación de la planta física. 


\section{La vista horizontal}

La estructura organizacional expuesta en este capítulo comprende un modelo vertical de una IES que sólo presenta las estructuras jerárquicas de la organización. Este modelo no muestra los productos (egresados, conocimiento, asesorías, etcétera) que son generados en una IES; tampoco los clientes a quienes se brindan servicios (empresas, mercado profesional, etcétera), y mucho menos, los flujos de trabajo (relaciones) que se dan en la IES para generar productos y servicios.

La vista horizontal de una organización (figura 2) ofrece una escenario más completo de la IES desde una perspectiva sistémica, la cual incluye a los tres elementos faltantes: los clientes, los productos y el flujo de trabajo (Rummler, 1991).

En este modelo es posible apreciar las unidades organizacionales descritas en el diagrama de estructura y la funcionalidad que en ellas se realiza, asimismo, las relaciones (flujos de trabajo) que se dan entre unidades organizacionales y con los clientes y proveedores de la IES. El modelo permite identificar a los proveedores de las IES: escuelas del nivel medio superior $y$ otras IES, las cuales entregan la materia prima (aspirantes) que es transformada en las divisiones académicas. Asimismo, se ve claramente a quién sirve una IES: empresas, el mercado profesional, y a la comunidad en general. Es a estas entidades a quienes la IES entrega los productos que genera.

Por último, se observa que cada unidad organizacional tiene a su cargo una serie de funciones cuyos flujos cruzan las fronteras de la unidad, estableciendo así los procesos que se realizan. Esta característica del modelo sustenta la premisa de la administración de procesos, es decir, las oportunidades para mejorar un proceso de negocio caen con frecuencia en estas interfaces (conocidas como flujos interfuncionales). Por ejemplo: la incorporación de programas y contenidos al procesos de enseñanza, una vez que se actualizan los planes de estudio; o también, la incorporación de aspirantes en el proceso de admisión, una vez que las instituciones de nivel medio superior concluyen un ciclo escolar.

Es así como las interfaces críticas, que permanecen ocultas en el modelo de la estructura organizacional, se hacen visibles en esta vista horizontal, permitiendo así el manejo de los procesos de una IES, no únicamente su organigrama. 
Arquitectura de procesos para las instituciones públicas de educación superior

\section{6}

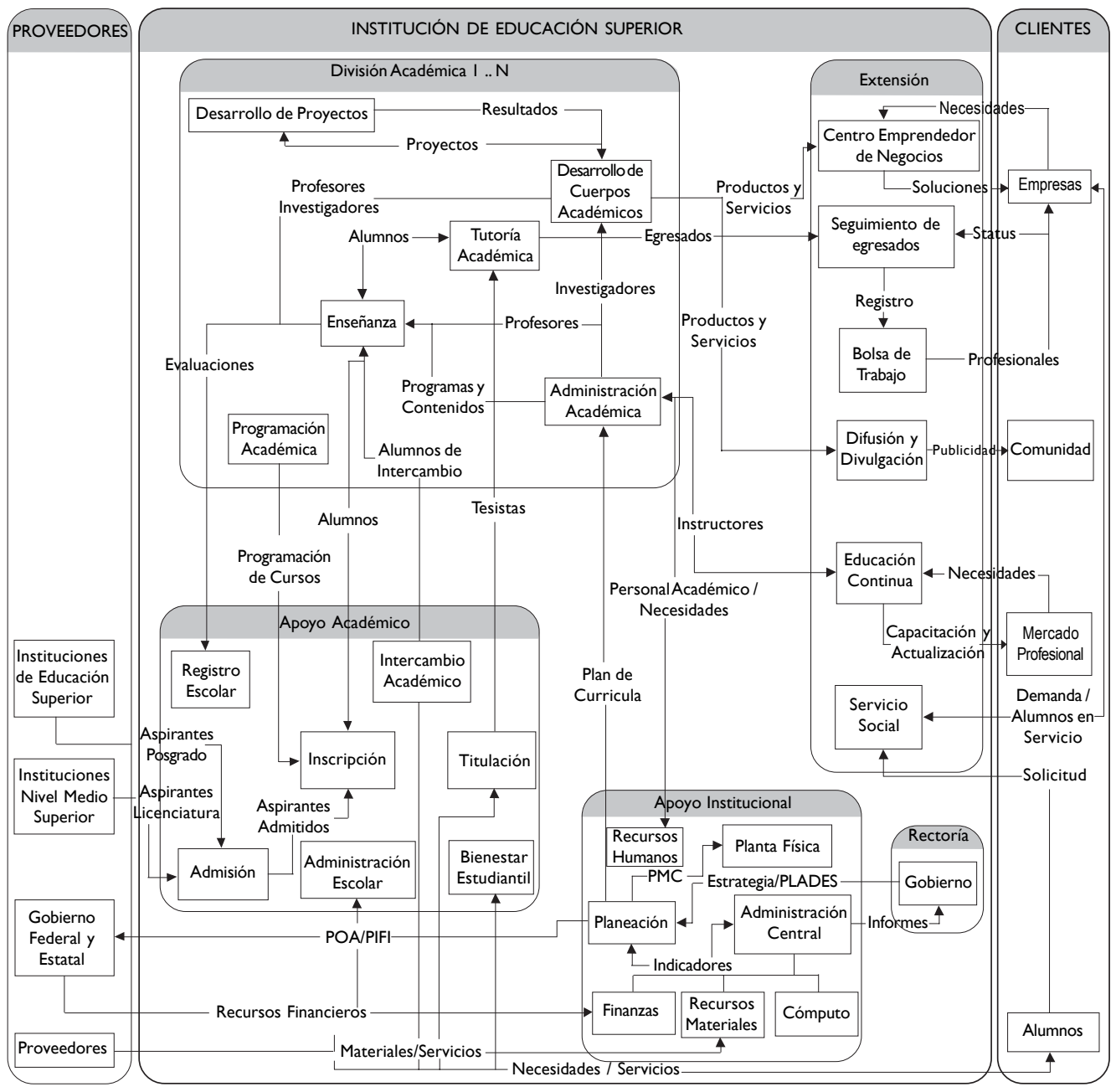

Figura 2. Vista Horizantal de una IES 


\section{La configuración de valor}

Una vez que se han identificado las actividades que comúnmente se realizan en una IES, éstas son clasificadas y agrupadas de acuerdo con el modelo de la Cadena de Valor (Porter, 2002), a través del cual es posible ver a una IES en función de las actividades que aportan valor a su tarea fundamental, la formación de profesionistas (figura 3). En este modelo se describen una serie de actividades que relacionan a los aspirantes a educación universitaria (egresados del nivel medio superior) con el mercado profesional, mediante el proceso enseñanza-aprendizaje (correspondiente al nivel superior).

El análisis de este modelo permite identificar actividades que pueden ser rediseñadas mediante la incorporación de tecnologías de información, incrementando así el valor de la organización. Se presentan las actividades encargadas de generar el valor en una IES, clasificadas de acuerdo con su responsabilidad (primarias o de apoyo) y la fase del proceso en la que son realizadas.

Partiendo de la premisa de que el proceso de enseñanza-aprendizaje (Docencia) es fundamental en toda IES, el modelo de la cadena de valor agregará todas las actividades que incrementan el valor de los productos y servicios resultantes de este proceso.

Entre las actividades de valor que se incluyen en este modelo, se destacan las correspondientes a la Enseñanza,Tutoría y Educación Continua, las cuales conforman el "core" de la función Docencia en las IES. También se puede observar que la mayor parte de las otras actividades incluidas en el modelo corresponden a la función adjetiva de apoyo académico. De acuerdo con lo anterior, se establece que el proceso de enseñanza-aprendizaje está sustentado en la función Docencia y fuertemente soportado por actividades de apoyo académico.

\section{La arquitectura de procesos de negocio}

La arquitectura de procesos es un modelo que nos presenta una idea general del quehacer de una organización; desglosa sus procesos fundamentales y las relaciones entre ellos y con otros procesos de soporte y administración.

El modelo de arquitectura de procesos (Maldonado, 2003) que se propone permite completar el modelo de negocios de una IES. Este modelo 
Arquitectura de procesos para las instituciones públicas de educación superior

\section{8}

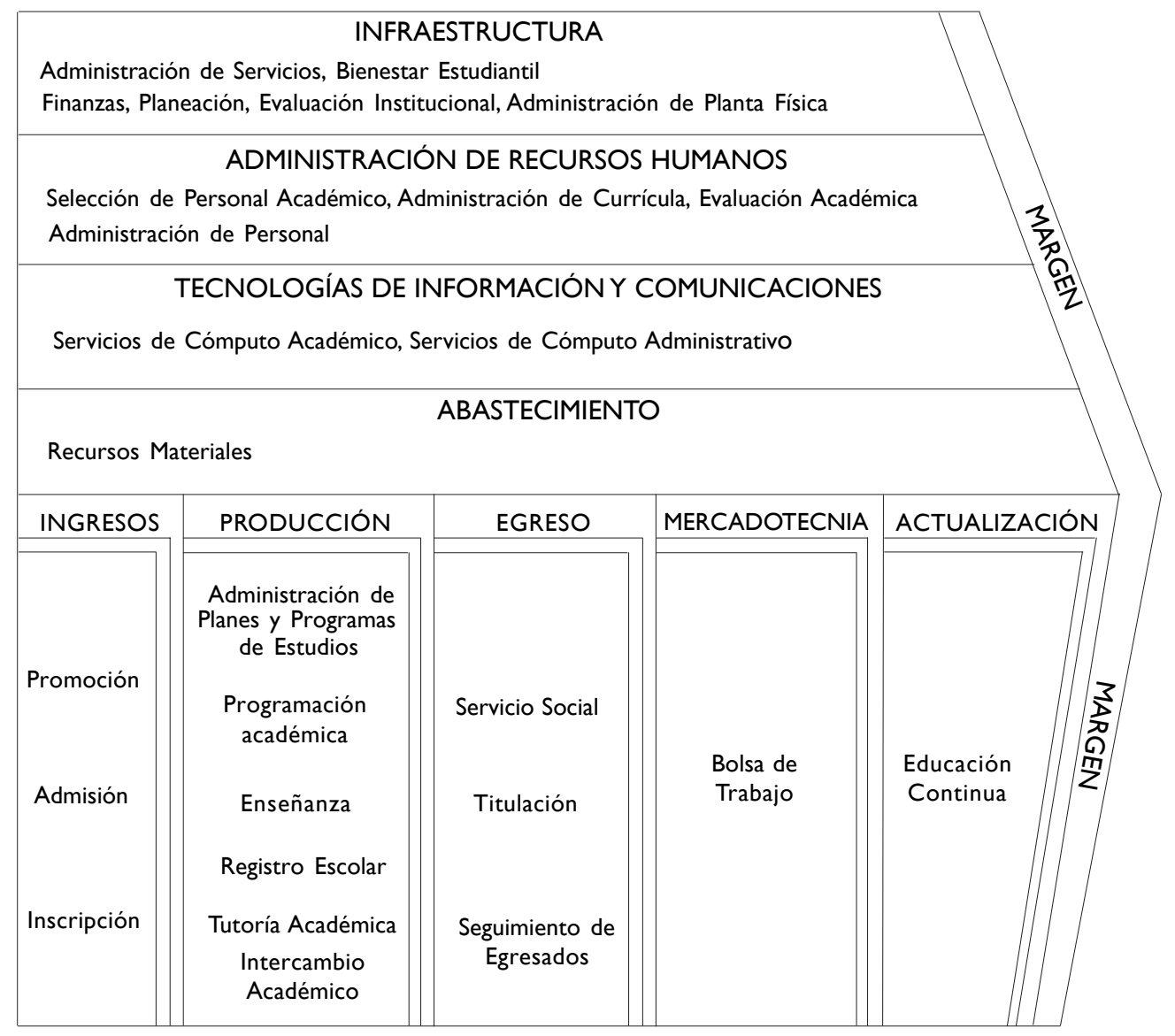

Figura 3. La Cadena de Valor de una IES 
complementa la forma en que se organiza la IES para realizar su trabajo (estructura organizacional); qué hace la IES, el trabajo necesario para generar estos productos, para quién lo hace (vista horizontal), así como las actividades que aportan valor a la IES (configuración de valor).

Este modelo parte de la definición de las funciones sustantivas y adjetivas de uso común en las IES, por lo que es posible caracterizar a una IES desde la perspectiva de sus procesos fundamentales: Docencia, Investigación, Extensión y Difusión; así como de sus procesos de apoyo:Apoyo Académico, Apoyo Institucional y Administración de Planta Física (figura 4).

\section{Proceso de Docencia}

Proceso fundamental en las IES que permite a los aspirantes, egresados de instituciones de los niveles medio superior y superior, acceder a una instrucción formal a través de programas académicos (oferta académica) diseñados para responder a los perfiles profesionales que demanda el mercado (empresas y organizaciones).

PROCESO de INVESTIGACIÓN

Comprende una serie de actividades encaminadas a la generación, aplicación y transmisión del conocimiento producido por investigadores organizados en cuerpos académicos y con base en una Línea General de Aplicación del Conocimiento (LGAC). La investigación se realiza en función de los programas académicos, y en respuesta a los requerimientos de empresas y organizaciones que son atendidos mediante consultorías y asesorías.

PROCESO DE EXTENSIÓN Y DIFUSIÓN

Las actividades de este proceso tienen el objetivo de entregar al entorno de la institución (empresas, organizaciones y comunidad) el producto final de sus procesos sustantivos de Docencia e Investigación: los profesionistas egresados de los distintos programas académicos de una IES son incorporados a las empresas (bolsa de trabajo); los conocimientos generados por los investigadores son difundidos y promovidos mediante acciones de extensión; los requerimientos de consultoría y asesoría especializada, provenientes del entorno de la institución, son identificados y canalizados a los cuerpos académicos correspondientes. 
Arquitectura de procesos para las instituciones públicas de educación superior

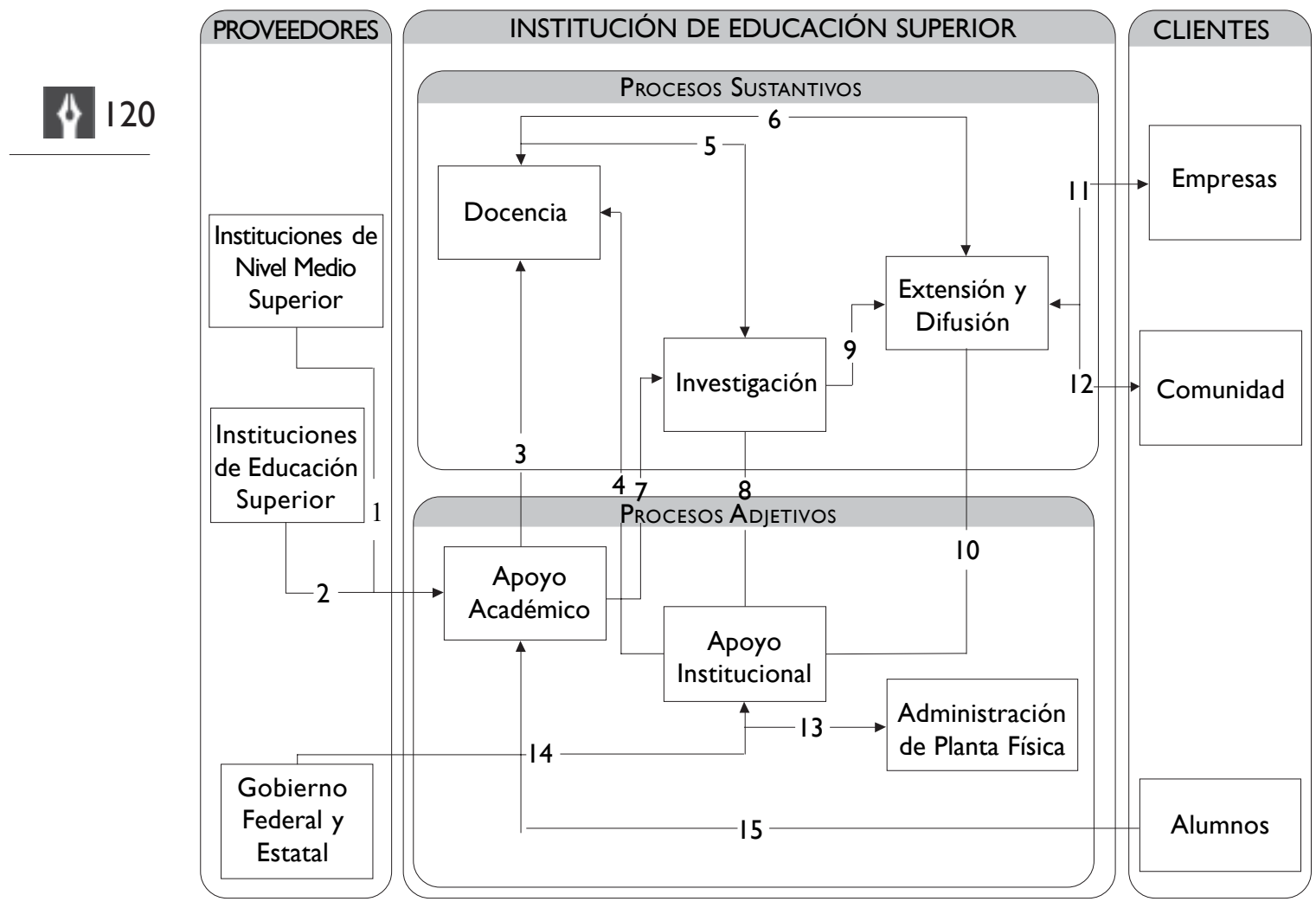

Figura 4. La Arquitectura de Procesos de una IES

Proceso deAporo ACADÉmico

Comprende las acciones encaminadas a soportar el proceso de enseñanza-aprendizaje en las IES: promoción, admisión, inscripción, registro escolar, programación académica, administración de planes y programas de estudio, servicio social, titulación, seguimiento de egresados, etcétera. Entre los productos más importantes generados en este proceso se encuentran: La base de datos con información académica de los alumnos, la base de datos que agrega los planes y programa de estudio y el historial de la oferta académica. 
Proceso deApoyo institucional

Este proceso atiende los requerimientos de las distintas unidades organizacionales de una IES: suministro de recursos materiales, la administración de personal académico y administrativo, la ejecución de procedimientos contables y financieros, la planeación, dirección, administración, evaluación y control de la institución. Entre los resultados más importantes que produce este proceso se encuentran, el registro del PIFI, del POA, la base de datos del personal académico y administrativo, la salvaguarda de los bienes de la institución, así como el suministro oportuno de recursos humanos, materiales y financieros necesarios para la operación de la institución.

Proceso de Administración de PLANTA FÍSICA

Mediante este proceso se define y ejecuta el plan de administración, operación, conservación, mantenimiento y expansión de la planta física. Las diversas unidades organizacionales de la IES generan una lista de requerimientos de espacios físicos (aulas, canchas, laboratorios, cubículos, oficinas, etc.) necesarios para realizar sus funciones. El producto de este proceso es la infraestructura de la institución.

El modelo que describe los procesos fundamentales y de apoyo de una IES, se presenta a continuación (véase figura 4). En este modelo podemos observar la relación que existe entre la institución y sus clientes y proveedores, así como las asociaciones entre los distintos procesos que conforman el "negocio" de una institución de educación superior.

La relación que se da entre estos procesos de negocio, y la que se establece con los clientes y proveedores de la institución queda descrita en los flujos de información siguientes:

I) Las Instituciones de Nivel Medio Superior generan y envían los insumos (egresados de los niveles 5 y 6 ) que dan inicio al proceso de Docencia.

2) Las Instituciones de Nivel Superior generan y envían los insumos (egresados de los niveles 7, 8 y 9) con que se inicia el proceso de Docencia.

3) El proceso de Docencia solicita a las áreas de Apoyo académico de la IES los insumos, procedimientos y servicios de apoyo académico 
Arquitectura de procesos

para las instituciones públicas

de educación superior

que requieren las actividades de Enseñanza, Tutoría y Educación Continua. El proceso de Apoyo académico, en respuesta a esta solicitud, realiza las actividades que van desde la admisión e inscripción, hasta la titulación y el seguimiento de egresados, a lo largo del proceso de Docencia.

4) El proceso de Docencia solicita a las áreas de Apoyo institucional de la IES los insumos, procedimientos y servicios de apoyo institucional que requieren las actividades de Enseñanza, Tutoría y Educación Continua. El proceso de Apoyo institucional suministra los recursos materiales, recursos humanos, procedimientos contables y financieros, líneas y objetivos estratégicos que se requieren en la realización del proceso de Docencia.

5) El proceso de Investigación incorpora profesores-investigadores en el proceso de Docencia, en apoyo a las actividades de Enseñanza, Tutoría y Educación Continua; asimismo, se incorporan alumnos en los proyectos de investigación.

6) El proceso de Docencia genera egresados y los envía al proceso de Extensión, a través del cual se abastece a las empresas que requieren profesionistas con el perfil correspondiente a los programas académicos de la IES y a los requerimientos del entorno.

7) El proceso de Investigación realiza actividades para generar conocimiento de acuerdo con las LGAC de los cuerpos académicos de la IES.

8) El proceso de Investigación solicita a las áreas de apoyo institucional de la IES los insumos, procedimientos y servicios de apoyo institucional que requieren las actividades inherentes a la generación de conocimiento. El proceso de Apoyo Institucional suministra los recursos materiales, recursos humanos, procedimientos contables y financieros, líneas y objetivos estratégicos que se requieren en estas actividades.

9) El proceso de Investigación envía sus productos generados (conocimiento con base en su LGAC) para su difusión, divulgación y aplicación, en atención a los requerimientos de empresas (asesorías y consultorías) y su entorno.

10) El proceso de Apoyo institucional suministra al proceso de 
Extensión y Difusión los recursos materiales y humanos, procedimientos contables y financieros, líneas y objetivos estratégicos, que requiere la divulgación del conocimiento.

II) Las unidades organizacionales a cargo del proceso de Extensión y difusión envían a las empresas y organizaciones del entorno de la IES, los profesionistas egresados de sus programas académicos y los resultados generados en respuesta a los servicios de consultoría y asesoría especializada.

12) El conocimiento generado por las actividades de investigación es entregado a la comunidad de la IES y a la sociedad en general.

13) La infraestructura de planta física de la IES es incorporada a los procesos sustantivos a través de las unidades organizacionales de apoyo administrativo.

14) Los gobiernos federal y estatal entregan a la IES los recursos financieros que requieren sus procesos sustantivos y adjetivos.

15) Los alumnos solicitan servicios de Apoyo académico al área correspondiente

\section{Conclusiones}

La arquitectura de procesos presentada comprende un enfoque sistémico de las funciones sustantivas y adjetivas que se realizan en toda IES. Este modelo proporciona una serie de recomendaciones que las IES pueden asumir para reordenar las actividades y tareas que en ellas se llevan a cabo.

El contar con una arquitectura de procesos permitirá conformar una base sólida para la construcción de servicios de información que soporten de manera eficiente los procesos de negocio de las instituciones de Educación Superior (IES).

La funcionalidad implícita (subprocesos) en cada uno de los procesos, el orden lógico (flujo de trabajo) en que estas funciones son realizadas, así como las actividades y acciones que comprende, conforman un marco de referencia conceptual para las IES. Este trabajo se presenta en la segunda parte de este artículo "La especificación de procesos en las IES", que se publicará en la siguiente edición de la revista Teoría y Praxis. 
Arquitectura de procesos para las instituciones públicas

de educación superior

\section{Fuentes consultadas}

Osterwalder, Alexander, Yves Pigneur (2002). And E-business Model Ontology for modelling e-Business, $15^{\text {th }}$ Bled Electronic Commerce Conference.

Henderson, J.C. and N.Venkatraman (1989). "Strategic Alignement: A framework for Strategic Information Technology Management”, CISR Working Paper No. 190, Massachusetts Institute of Technolog,.

Rummler, Geary A. and Alan P. Brache (1991). "The Horizontal View. Managing The White Space". The Magazine of Human resources Development.

Porter, Michael E. (2002) Ventaja Competitiva. CECSA.

Maldonado, Armando (2003). Arquitectura de Procesos. Curso de Arquitectura de la Empresa, ITAM. 